\title{
Using A Multi-Agent System Approach for Monitoring Appropriateness Criteria in Medical Imaging
}

\author{
Mahtab Karami ${ }^{1, *}$ \\ ${ }^{1}$ Kashan University of Medical \\ "Corresponding author: Mahtab Karami, Kashan University of Medical. E-mail: Skarami_m@kaums.ac.ir
}

Received 2016 December 21; Accepted 2017 February 08.

\begin{abstract}
Agent technology is an emerging and promising research area in software technology, which increasingly contributes to the development of value-added information systems for large healthcare organizations due to its capability to automatically modify themselves in response to changes in their operating environment. Multi agent system (MAS) approach is the construction of a complex system as a set of entities, called agents, interacting among them in order to perform the system tasks. Patie Maes, the director of MIT media lab, deems that agent is "a computer system, which locates in dynamic and complex environment, can autonomically sense the environment and act accordingly to complete its tasks or goals". Agent is a computing entity with four features of autonomy, reactivity, interaction and initiative. A MAS is composed of a large number of agents and other computational artifacts. These agents are goal-oriented components, that is, they are modeled as entities that pursue goals and choose for execution those actions that will potentially contribute to satisfy them. These choices depend on their information about the environment, past experiences and themselves. Agents are also social because they need to interact with other agents to achieve the satisfaction of goals, and these interactions are modeled in terms of information, requests and informs. In healthcare, medical imaging is a service-oriented, dataintensive and technology-driven environment; therefore it requires combining many computerized systems with different user environments to provide quality efficient services in less time. In such environments, MAS is well-suited to describe the interactions between agents (e.g., information exchanged, related tasks or reasons for that interaction) and the way in which the agent adapts to changes in it (e.g., new roles or goals) or its environment. Medical imaging is a science but also a discipline of action that often requires a decision. The complexity of decision-making, especially in medical imaging, comes from the uncertainty, for example the uncertainty of knowledge, uncertainty about the protocols and the uncertainty of the procedure used. A multi-agent system (MAS) for decision-aiding support uses and combines distributed and heterogeneous systems to ease the extraction of useful information in order to select appropriate protocol or procedure.
\end{abstract}

Keywords: Medical Imaging, Agent Technology, Multi Agent System, Agent, Multi Agent System, Agent, Appropriateness Criteria

This is an abstract presented in the 33rd Iranian congress of radiology (ICR) and the 15th congress of Iranian radiographic science association (IRSA). 